\title{
Neutrino searches at the Pierre Auger Observatory
}

\author{
S. Pastor ${ }^{\mathrm{a}}$, for the Pierre Auger Collaboration ${ }^{\mathrm{b}}$ \\ ${ }^{a}$ Institut de Física Corpuscular (CSIC-Universitat de València), Apartado de Correos 22085, 46071 Valencia, Spain \\ ${ }^{b}$ Observatorio Pierre Auger, Av. San Martín Norte 304, 5613 Malargüe, Argentina \\ (Full author list: http://www.auger.org/archive/authors_2012_07.html)
}

\begin{abstract}
The surface detector array of the Pierre Auger Observatory is sensitive to ultra-high energy neutrinos in the cosmic radiation. Neutrinos can interact in the atmosphere close to ground (down-going) and, for tau neutrinos, through the Earth-skimming mechanism (up-going) where a tau lepton is produced in the Earth crust that can emerge and decay in the atmosphere. Both types of neutrino-induced events produce an inclined particle air shower that can be identified by the presence of a broad time structure of signals in the water-Cherenkov detectors. We discuss the neutrino identification criteria used and present the corresponding limits on the diffuse and point-like source fluxes.
\end{abstract}

Keywords: Pierre Auger Observatory, Ultra-high energy neutrinos

\section{Introduction}

The existence of cosmic neutrinos with energies in the $\mathrm{EeV}$ range and above is required by the observation of ultra-high energy cosmic rays (UHECRs). Although the nature of the very energetic cosmic radiation and its production mechanisms are still uncertain $[1,2]$, all models of UHECRs predict neutrino fluxes from the decay of charged pions, produced in UHECR interactions either in their sources or with background radiation fields, such as the cosmogenic or GZK neutrinos [3], produced in the interaction of UHE protons with the cosmic microwave background. Their flux is to some extent uncertain because it depends on the composition of primary UHECRs and on the nature, cosmological evolution and spatial distribution of the sources [4].

The observation of UHE neutrinos would open a new window to the universe, giving information on regions that are otherwise hidden from observation by large amounts of matter in the field of view. Moreover, neutrinos travel unaffected by magnetic fields, essentially maintaining the direction of their production sites. Their detection is the aim of many experiments which employ different techniques, from neutrino telescopes such as IceCube or Antares to experiments like ANITA, that searches for radio waves from extra-terrestrial neutrino interactions. One of the detection techniques is based on the observation of extensive air showers (EAS) in the atmosphere initiated by UHE neutrinos, which could be detectable by a large ground-based detector.

In this contribution, we describe the sensitivity of the surface detector array of the Pierre Auger Observatory to UHE neutrinos with energies around $\mathrm{EeV}$ and larger. We explain the identification criteria used to distinguish neutrino-induced showers from those initiated by UHECRs, such as protons or heavy nuclei. The analysis of Auger data reveals no neutrino candidates, leading to stringent limits on the flux of UHE neutrinos.

\section{Detection of UHE neutrinos with the Pierre Auger Observatory}

Neutrinos, even at very high energies, present a low interaction probability which means that a large amount of matter is needed to detect these elusive particles. In the case of UHE cosmic neutrinos, the Earth atmosphere is the target where the primary particles interact producing an EAS detectable in experiments such as the Pierre Auger Observatory. These are the so-called down-going 


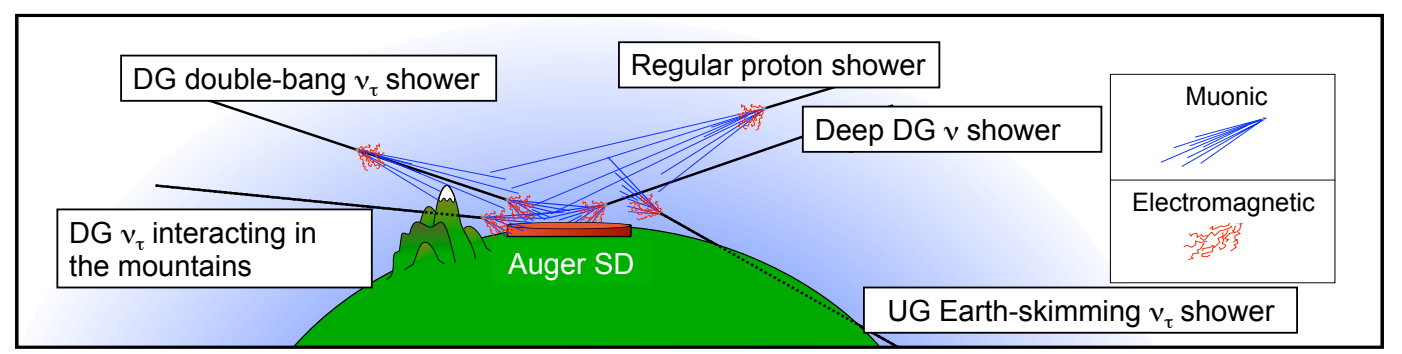

Figure 1: Simplified picture of the different types of particle showers induced by UHE neutrinos compared to proton-induced showers. The surface detector of the Pierre Auger Observatory is sensitive to both up-going or Earth-skimming tau neutrinos and down-going neutrinos of all flavours.

(DG) events, where neutrinos of all flavours interact at any atmopheric depth through charged-current (CC) or neutral-current (NC) weak processes and develop an EAS. Instead, only UHE tau neutrinos can lead to upgoing (UG) or Earth-skimming events in an efficient way, when they interact in the Earth crust and produce a tau lepton that can emerge and decay in the atmosphere. If such decays occur in flight over the detector array, they may initiate detectable EAS $[5,6]$. Tau neutrinos are expected to be suppressed in the production processes, but the effect of flavour neutrino oscillations over cosmological distances modifies the initial composition and leads to approximately equal fluxes for all flavours. DG and UG neutrino-induced showers are depicted in Fig. 1.

The main background for the detection of neutrinoinduced EAS is the particle showers initiated by protons or heavy nuclei and possibly photons. UHECRs interact high in the atmosphere, producing EAS that contain muons and an electromagnetic (EM) component of electron, positrons and photons. This latter component reaches a maximum at an atmospheric depth of order $800 \mathrm{~g} \mathrm{~cm}^{-2}$, extinguishing gradually within the next $1000 \mathrm{~g} \mathrm{~cm}^{-2}$. Thus after roughly a couple of vertical atmospheric depths only high-energy muons survive. In the first stages of development the time spread of the particles in the shower front is large $(\sim \mu \mathrm{s})$. When the shower becomes old, most of the particles in the shower front, the high-energy muons, arrive in a short time window ( $100 \mathrm{~ns})$. As a consequence very inclined showers induced by UHECRs in the upper atmosphere reach the ground as a thin and flat front of muons accompanied by an EM halo (produced by bremsstrahlung, pair production, and muon decays), with a time structure very similar to that of the muons. Instead, if a shower is induced by a particle that interacts deep in the atmosphere (a $v$ interaction in air, or a tau decay), its EM component could hit the ground and give a distinct broad signal in time. The panels in Fig. 2 represent these various cases.

The Pierre Auger Observatory [7] was designed to measure UHECRs with unprecedented precision. It employs a hybrid technique to detect EAS: arrays of surface particle detectors (SD) and telescopes that detect fluorescence radiation. The SD array of the Auger Observatory in the Mendoza province (Argentina), consists of 1600 water Cherenkov tanks arranged in a triangular grid of $1.5 \mathrm{~km}$ that covers an effective area of $3000 \mathrm{~km}^{2}$. Each cylindrical tank of $10 \mathrm{~m}^{2}$ surface contains purified water, $1.2 \mathrm{~m}$ deep, and is instrumented with three 9" photomultiplier tubes (PMT) sampled by $40 \mathrm{MHz}$ Flash Analog Digital Converters (FADCs). Each tank is regularly monitored and calibrated in units of vertical equivalent muon (VEM) corresponding to the signal produced by a muon traversing the tank vertically [8].

The signal in each SD station is digitized using FADCs with a $25 \mathrm{~ns}$ time resolution, which allows distinction between the narrow signals induced by muons and the broad signals from the EM component. Thus the time structure and shape of the FADC traces, characterized by several observables, can help us to discriminate stations hit by an EAS in the early stages of development or by an old shower. A set of conditions has been designed and optimized to select showers induced by UHE neutrinos, which constitute the neutrino identification criteria for either UG or DG events, rejecting those induced by UHECRs. The fluorescence detectors can also be used for neutrino searches but the nominal $10 \%$ duty cycle of this technique reduces the sensitivity. Here only the data collected with the SD of the Pierre Auger Observatory is used to search for UHE neutrinos.

\section{Neutrino identification criteria}

A large set of simulations of UHE neutrinos forced to interact deep in the atmosphere were produced in order to characterize the signal that their induced EAS would 


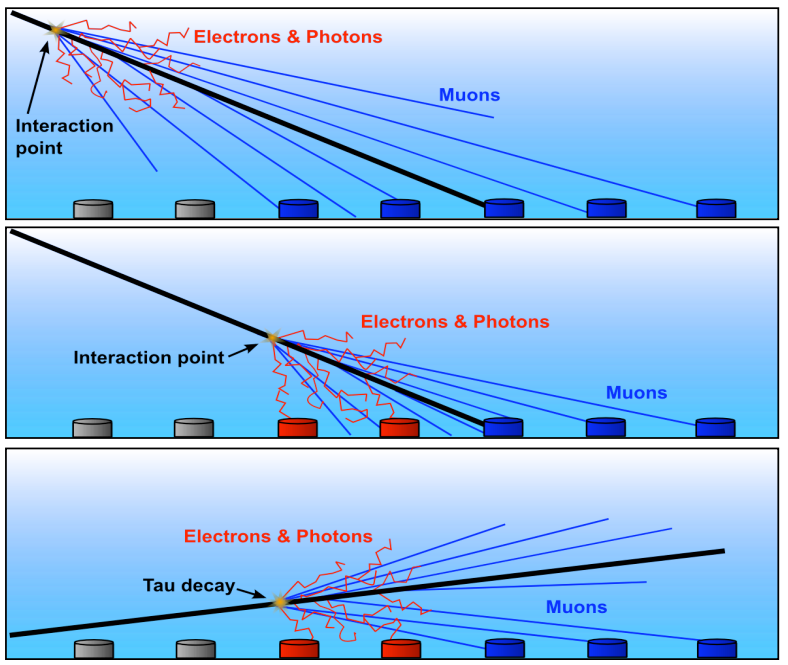

Figure 2: Upper panel: an inclined EAS induced by a proton interacting high in the atmosphere. The EM component is absorbed and only the muons reach the array of ground detectors. Middle panel: a primary UHE neutrino can initiate a deep inclined shower whose early region has a significant EM component at the detector level. Lower panel: a "young" shower can be also produced by an up-going tau lepton produced by the interaction of an Earth-skimming tau neutrino.

produce at the SD array. The first NC or CC interaction of the primary neutrino in the air was simulated using HERWIG [9], whereas the AIRES code [10] was used for the EAS development. For $v_{\tau}$-induced showers, the TAUOLA package [11] was used to simulate the $\tau$ decay and obtain the secondary particles and their energies.

Monte Carlo (MC) neutrino shower simulations were performed including the geographic conditions of the Auger site (e.g. geomagnetic field) for different zenith angles. For UG showers, primary energies ranged from $10^{17}$ to $3 \times 10^{20} \mathrm{eV}$ at zenith angles between $90.1^{\circ}$ and $95.9^{\circ}$ and at an altitude of the decay point above the Observatory up to $2500 \mathrm{~m}$. The propagation through the Earth's crust of up-going $v_{\tau}$ 's was simulated. For DG neutrinos, simulations were performed at $\theta=75^{\circ}, 80^{\circ}, 85^{\circ}, 87^{\circ}, 88^{\circ}$ and $89^{\circ}$, random azimuth angles between $0^{\circ}$ and $360^{\circ}$ and different hadronic models. The primary neutrinos were forced to interact at different slant depths measured from the ground up to a maximum value depending on $\theta$. Finally the response of the SD array is simulated using the Offline package [12]. The sets of UG and DG simulations were used to estimate the expected signal and the detection efficiency.

The main criterion to identify young and very inclined showers consists of looking for broad time signals in the SD stations. A different set of identification conditions was designed for UG and DG $v$ searches.

\subsection{Up-going neutrinos}

Young showers are expected to trigger detector stations with broad signals releasing a so-called Time Over Threshold (ToT) trigger [13]. Counting ToTs stations can help identifying young showers, and a cut in the value of the area of the signal over its peak (AoP, where the peak corresponds to the maximum measured current of recorded trace at a single water-Cherenkov detector) is applied to reject accidental muons hitting a station that could mimic a ToT local trigger. After trace cleaning, very inclined showers are identified with the elongation of their footprint, defined by the ratio of length (L) over width $(\mathrm{W})$ of the shower pattern on ground, requiring $L / W>5$, and the mean apparent velocity $\langle V\rangle$, expected to be compatible with the speed of light for quasi-horizontal showers, in the range $(0.29,0.31) \mathrm{m}$ $\mathrm{ns}^{-1}$ with an r.m.s. scatter below $0.08 \mathrm{~m} \mathrm{~ns}^{-1}$. Finally compact configurations of selected ToTs complete the expected picture of young $v_{\tau}$-induced shower footprints. For more details, we refer the reader to refs. $[14,15]$.

\subsection{Down-going neutrinos}

DG neutrino events are also young and inclined showers, but the wider range of zenith angles requires different identification criteria, as described in [16]. For this purpose data collected with the Auger SD between 1 Jan 2004 and 31 Oct 2007 ( 1.2 years of the full SD array) was used as "training" data. Showers that trigger the SD array but arrived during periods with instabilities in data acquisition are excluded. After that the FADC traces are cleaned to remove segments that are due to accidental muons not belonging to the shower but arriving close in time with the shower front. Moreover, if 2 or more segments with areas comparable to each other appear in a trace the station is classified as ambiguous and it is not used. Then a selection of the stations actually belonging to the event is done based on space-time compatibility. Events with less than 4 tanks passing the level 2 trigger algorithm [7] are rejected. This sample is then searched for inclined events requiring that the triggered tanks have elongated patterns on the ground, with a cut $L / W>3$. The average speed $\langle V\rangle$ measured between pairs of triggered stations is required to be compatible with that expected in a simple planar model of the shower front in an inclined event with $\theta \geq 75^{\circ}$, allowing for some spread due to fluctuations $(\langle V\rangle \leq 0.313$ $\left.\mathrm{m} \mathrm{ns}^{-1}\right)$. Only events with reconstructed zenith angle $\theta \geq 75^{\circ}$ are selected. Exactly the same set of conditions is applied to the simulated neutrinos.

The sample of inclined events is searched for young showers using observables characterizing the time duration of the FADC traces in the early region of the 


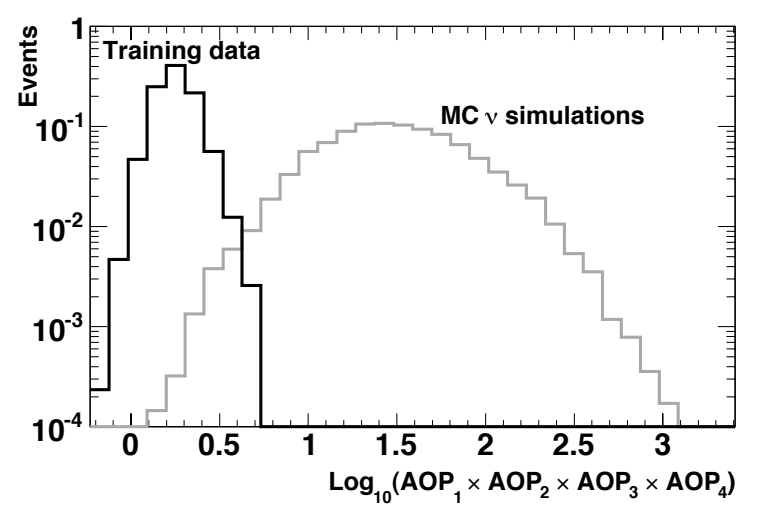

Figure 3: Distribution of the product of the AoP of the first four triggering stations in background (real events in the training sample) and simulated $v_{e}$ charged-current events.

event. To optimize their discrimination power we apply the Fisher method [17] to the training data, dominated by nucleonic showers, and to the MC simulations of neutrino-induced showers. Given two populations of events characterized by a set of observables, the Fisher method produces a linear combination of the various observables (the Fisher discriminant $f$ ) so that the separation between the means of $f$ in the two samples is maximized, whereas the quadratic sum of the r.m.s. of $f$ in each of them is minimized. Because SD events with a large multiplicity $N$ (number of tanks) are different from events with small $N$, the sample of training data is divided into 3 sub-samples (events with $4 \leq N \leq 6,7 \leq N \leq 11$ and $N \geq 12$ ). We use 10 discriminant variables of the Fisher estimator: the AoP and its square of the first 4 tanks in each event, their product, and a global early-late asymmetry. In Fig. 3 one can see a clear separation between the distributions of one of these variables for neutrino simulated showers and real inclined events.

In Fig. 4 we present the distribution of the Fisher discriminant for the training data and DG neutrino simulations with multiplicity $7 \leq N \leq 11$. Again both samples are well separated. The expected number of background events can be computed by extrapolating the exponential tail of the data distribution. This allows us to find a cut-off value $f_{\text {cut }}$ for each of the sub-samples, so that we expect less than one background event every 20 years above its value. Events with $f>f_{\text {cut }}$ are considered to be neutrino candidates. These cuts reject all real events in the training data samples while keeping a significant fraction of the MC neutrino simulations [16].
Fisher distribution - medium mult. $(7 \leq \mathrm{N} \leq 11)$

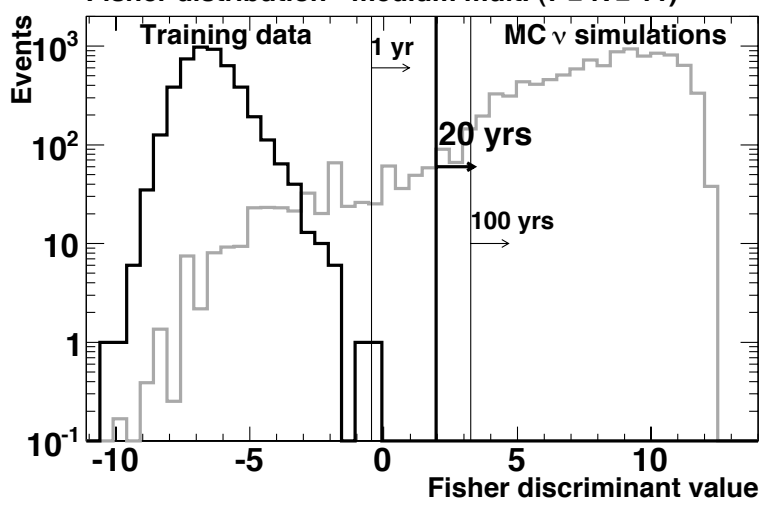

Figure 4: Distribution of the Fisher discriminant for events with station multiplicity $7 \leq N \leq 11$, for real Auger data in the training period (1 Jan 2004 - 31 Oct 2007) and MC simulated DG neutrinos. The vertical lines indicate the cut in the Fisher value that needs to be placed to have less than 1 event in each period of time (1, 20 or 100 years).

\section{Exposure and limits on UHE neutrinos}

Auger data have been analyzed to look for candidate events that fulfilled the selection criteria for Earthskimming UHE tau neutrinos, as described in [14, 15]. This analysis has been updated with data equivalent to 3.5 years of the full Auger SD in [18]. In [16] we describe the selection procedure and values of $f_{\text {cut }}$ for the identification of DG neutrino events to real data from 1 Nov 2007 to 31 May 2010 ( 2.0 years of the full SD array), i.e. after the training period mentioned above. Over the period analyzed, no candidate events were found for either UG or DG neutrinos. Based on this, the Pierre Auger Observatory data can be used to place the corresponding limits on the diffuse flux of UHE neutrinos. For this purpose the total exposure of the Auger SD must be evaluated, which involves folding the SD array aperture with the interaction probability and the identification efficiency, and integrating in time taking into account changes in the array configuration due to the installation of new stations and instabilities in data taking.

For both UG and DG neutrinos, the identification efficiency depends on the position of the shower in the surface covered by the array and the time through the instantaneous configuration of the array. The efficiency for UG neutrinos is also a function of the $\tau$ energy and the altitude above ground of the central part of the shower $h_{c}$ (defined at $10 \mathrm{~km}$ after the decay point [19]). Instead, for DG neutrinos the efficiency depends on the primary energy and the depth along the atmosphere at 


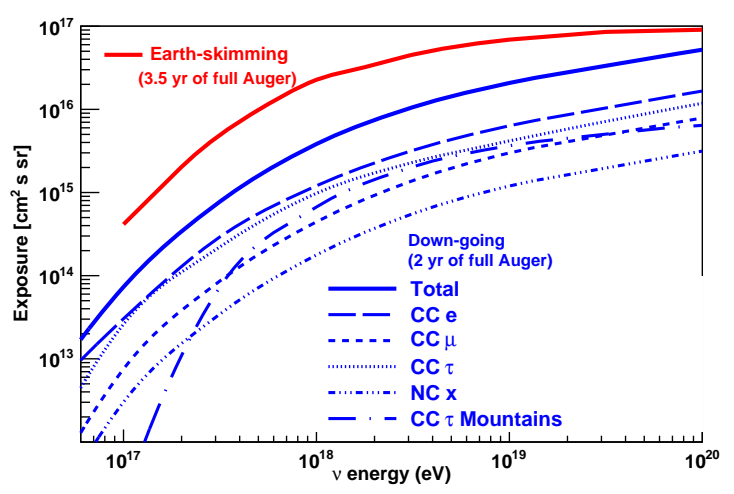

Figure 5: Exposure of the surface detector of the Pierre Auger Observatory for Earth-skimming and DG neutrino showers.

which the neutrino interacts, as well as on its flavour and type of interaction (CC or NC), because the different combinations of both induce different types of showers. The efficiencies for each case were obtained through MC simulations of the development of the shower in the atmosphere and the simulation of the surface detector array.

The Auger exposure to UHE neutrinos, $\varepsilon\left(E_{v}\right)$, shown in Fig. 5, was calculated using purely MC techniques and integrating the neutrino identification efficiencies over the whole parameter space $[14,15,16]$. Several sources of systematic uncertainties have been taken into account and their effect on the exposure evaluated. Here we do not include the full list, but we note that the main systematic uncertainty is the calculation of $\tau$ energy losses for UG neutrinos [15] and the neutrino cross section for DG.

Once the exposure has been calculated, a limit on the UHE $v$ flux can be obtained assuming a known shape. For a $f(E)=k \cdot E^{-2}$ differential neutrino flux we have obtained a limit on the single flavour neutrino flux using DG showers [16]

$$
k<1.7 \times 10^{-7} \mathrm{GeV} \mathrm{cm}^{-2} \mathrm{~s}^{-1} \mathrm{sr}^{-1} \text { (90\% C.L.) }
$$

The corresponding limit on Earth-skimming $v$ 's is [18]

$$
k<3.2 \times 10^{-8} \mathrm{GeV} \mathrm{cm}^{-2} \mathrm{~s}^{-1} \mathrm{sr}^{-1} \text { (90\% C.L.), }
$$

which updates the limits in $[14,15]$. Both limits are shown in Fig. 6, compared with the bounds from the IceCube [20] and ANITA [21] experiments. We also include in Fig. 6 the Auger limits in differential format, calculated as $2.44 / E_{v} \varepsilon\left(E_{v}\right)$, in order to emphasize the energy range at which the sensitivity of the Pierre Auger Observatory to UHE neutrinos peaks. The shaded area

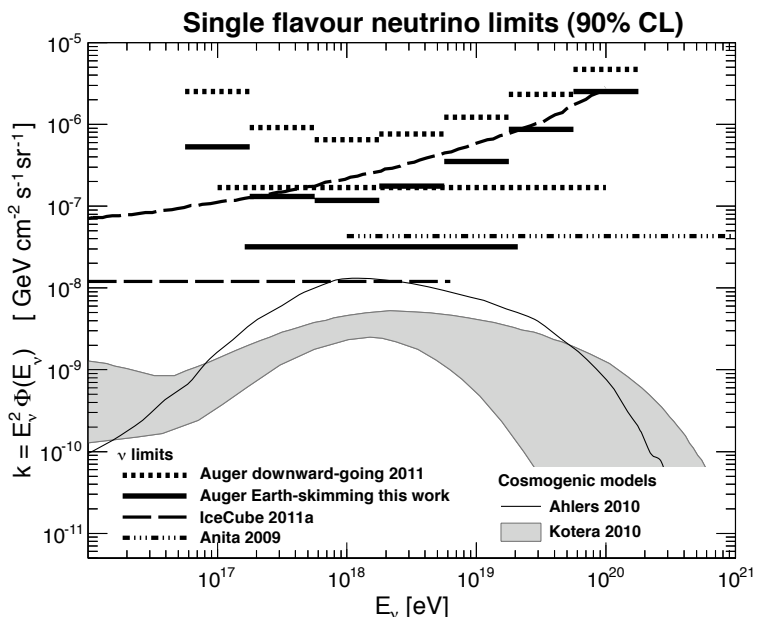

Figure 6: Integrated (horizontal solid lines) and differential (segments) upper limits ( $90 \%$ C.L.) on the single flavour $E_{v}^{-2}$ flux of UHE neutrinos, as a function of the neutrino energy. Limits from the Pierre Auger Observatory (down-going and Earth-skimming) and the IceCube and ANITA experiments are shown, in comparison with predictions for cosmogenic neutrinos under different assumptions [22, 23].

in Fig. 6 brackets the cosmogenic neutrino fluxes predicted under a wide range of assumptions in [22], and the flux computed in [23] and consistent with HiRes and Fermi-LAT measurements is also shown.

\section{Limits to point-like sources}

We saw in section 3 that the neutrino search at the Pierre Auger Observatory is limited to highly inclined showers. Thus, at each instant, neutrinos can be detected only from a specific portion of the sky, limited by the zenith angle ranges for the UG and DG analyses. The sensitivity of the Auger SD to point-like sources of UHE neutrinos depends on its declination, approximately spanning north of $-65^{\circ}$ and south of $55^{\circ}$. As an example, the active galaxy Centaurus A (at a declination $\left.-43^{\circ}\right)$ is observed $\sim 7 \%(\sim 15 \%)$ of one sidereal day in the range of zenith angles corresponding to the UG (DG) search, whereas the regions close to the Northern and Southern Terrestrial Poles are not accessible.

The exposure of the SD as a function of the neutrino energy and of the source position in the sky is evaluated with an identical procedure to that used for the calculation for a diffuse flux of UHE neutrinos, with the exception of the solid angle integration over the sky [18]. Assuming a $f(E)=k_{\mathrm{PS}} \cdot E^{-2}$ differential neutrino flux, the corresponding limit to $k_{\mathrm{PS}}$ is derived as a function of 


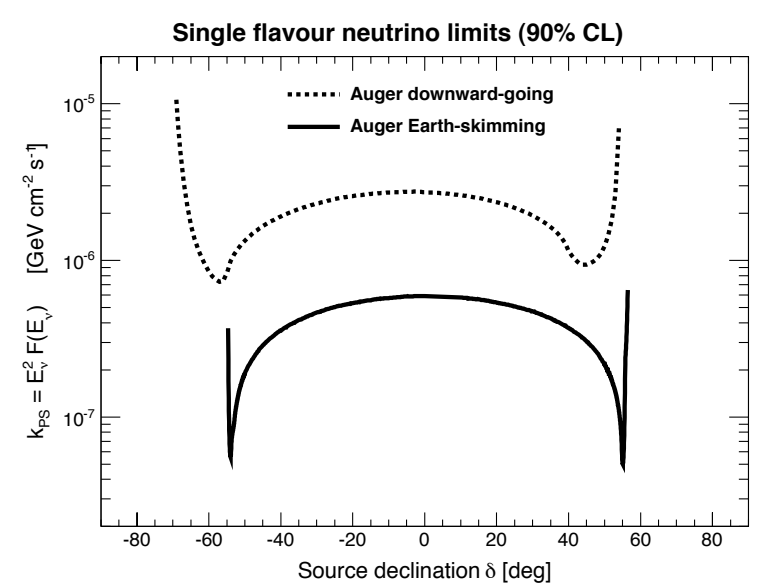

Figure 7: Upper limits at $90 \%$ C.L. on a single flavor $E_{v}^{-2}$ flux from a specific point-like source as a function of its declination. The bounds from the Earth-skimming and downward-going neutrino analyses hold for a neutrino energy range $10^{17}-10^{20} \mathrm{eV}$.

the source declination. The $90 \%$ C.L. upper limits from the UG and DG analyses are shown in Fig. 7, whereas the upper bounds on $k_{\mathrm{PS}}$ for the particular case of Centaurus A, a potential acceleration site for UHECRs, are shown in Fig. 8 together with the predicted fluxes of two theoretical models $[26,27]$ of UHE neutrino production.

\section{Conclusions}

The surface detector array of the Pierre Auger Observatory is sensitive to the EAS initiated by UHE neutrinos in the atmosphere, either down-going or Earthskimming neutrinos. We have shown how using MC simulations and training data the identification criteria for UHE neutrinos can be found. The data collected by the Auger SD has been used to present upper limits to the flux of UHE neutrinos at EeV energies, both diffuse and from point -like sources. The Pierre Auger Observatory will keep taking data over the coming years to significantly improve the bounds if no neutrino candidate is found.

\section{Acknowledgments}

Work supported by the Spanish grants FPA201122975 and CSD2009-00064 (MINECO), and PROMETEO/2009/091 (Generalitat Valenciana).

\section{References}

[1] F. Halzen and D. Hooper, Rep. Prog. Phys. 65 (2002) 1025

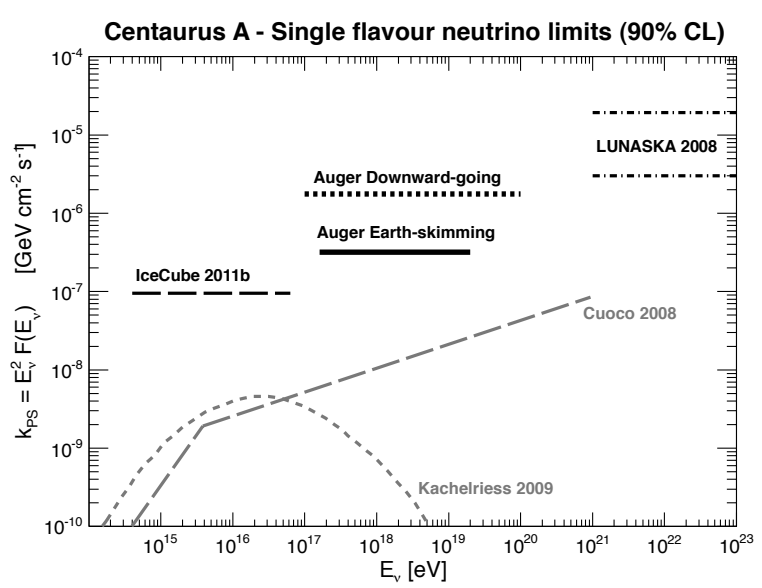

Figure 8: Upper limits at $90 \%$ C.L. on a single flavor $E_{v}^{-2}$ flux from the active galaxy Centaurus A from the Earth-skimming and downwardgoing neutrino analyses, together with bounds from the IceCube Neutrino Observatory [24] and LUNASKA [25]. The predictions for two models of UHE $v$ production $[26,27]$ are also shown.

[2] P. Bhattacharjee and G. Sigl, Phys. Rep. 327 (2000) 109

[3] V. S. Berezinsky et al., Phys. Lett. B 28 (1969) 423

[4] L. A. Anchordoqui et al., Phys. Rev. D 76 (2007) 123008

[5] D. Fargion, Astrophys. J. 570 (2002) 909

[6] A. Letessier-Selvon, AIP Conf. Proc. 566 (2001) 157

[7] J. Abraham et al. [Pierre Auger Coll.], Nucl. Instr. and Meth. A 523 (2004) 50

[8] X. Bertou et al. [Pierre Auger Coll.], Nucl. Instr. and Meth. A 568 (2006) 839

[9] G. Corcella et al., JHEP 01 (2001) 010

[10] S. J. Sciutto, preprint arXiv:astro-ph/9911331

[11] S. Jadach et al., Comput. Phys. Commun. 76 (1993) 361

[12] S. Argirò et al., Nucl. Instrum. Meth. A 580 (2007) 1485

[13] P. Billoir and O. Blanch, Nucl. Phys. B Proc. Suppl. 168 (2007) 225

[14] J. Abraham et al. [Pierre Auger Coll.], Phys. Rev. Lett. 100 (2008) 211101

[15] J. Abraham et al. [Pierre Auger Coll.], Phys. Rev. D 79 (2009) 102001

[16] P. Abreu et al. [Pierre Auger Coll.], Phys. Rev. D 84 (2011) 122005

[17] R. Fisher, Annals Eugen. 7 (1936) 179

[18] P. Abreu et al. [Pierre Auger Coll.], Astrophys. J. Lett. 755 (2012) L4

[19] X. Bertou et al., Astropart. Phys. 17 (2002) 183

[20] R. Abbasi et al. [IceCube Coll.], Phys. Rev. D 83 (2011) 092003

[21] P. W. Gorham et al. [ANITA Coll.], Phys. Rev. D 82 (2010) 022004

[22] K. Kotera, D. Allard and A. V. Olinto, JCAP 10 (2010) 013

[23] M. Ahlers et al., Astropart. Phys. 34 (2010) 106

[24] R. Abbasi et al. [IceCube Coll.], Astrophys. J. 732 (2011) 18

[25] C. W. James et al. [LUNASKA Coll.], MNRAS 410 (2011) 885

[26] A. Cuoco and S. Hannestad, Phys. Rev. D 78 (2008) 023007

[27] M. Kachelriess, S. Ostapchenko and R. Tomàs, New J. Phys. 11 (2009) 065017 